\title{
AGE-RELATED DYNAMICS IN POST-VACCINE ANTIBODY IMMUNE RESPONSE TO DIPHTHERIA AND TETANUS TOXOID IN BULGARIAN SUBJECTS
}

\author{
S. Lesichkova ${ }^{1,2}$, S. Mihailova ${ }^{1,2}$, A. Mihaylova ${ }^{1}$, N. Gesheva1 ${ }^{1}$, P. Yankova ${ }^{1,2}$, E. Naumova ${ }^{1,2}$ \\ ${ }^{1}$ Department of Clinical Immunology and Stem Cell Bank, University Hospital Alexandrovska, \\ ${ }^{2}$ Medical Faculty, Medical University - Sofia, Bulgaria
}

\begin{abstract}
In this study, we investigated the age-related dynamics in post-vaccine humoral immunity to diphtheria (DT) and tetanus (TT) toxoids in the Bulgarian population. In addition, we attempted to correlate the titers of specific antibodies with the predisposition to more common infectious pathology among our study participants. The 208 individuals tested were divided into five age groups: 0-4, 4-6, 6-12, 12-17 and 17-66 years, based on the vaccines received according to the immunization schedule in Bulgaria. Vaccine response was determined by measuring the concentrations of specific IgG antibodies using commercial ELISA kits. Sufficient protective levels of diphtheria $(>0.1 \mathrm{IU} / \mathrm{mL}$ ) and tetanus (>0.15 IU/mL) antitoxin were detected in $63.5 \%$ and $85.1 \%$ of all subjects, respectively. The highest rates of protection against both TT (94.3\%) and DT (79.2\%) were observed in the youngest age group (0-4 years). We also observed a relatively high rate of insufficient protection $(<0.1 \mathrm{IU} / \mathrm{ml})$ against diphtheria $(36 \%$ of individuals tested across all age groups) in comparison to tetanus (14.9\% of all subjects). The rate of insufficient protection against both antigens was higher among children with frequent infections. Moreover, $77.1 \%$ of the individuals having low antibody titers against the highly immunogenic tetanus toxoid, also had low levels of diphtheria antibodies. The level of seroprotection is better for tetanus than for diphtheria toxoid at any age. In conclusion, our data provide information on the level of immunity to diphtheria and tetanus among vaccinated individuals in Bulgaria and allows for the identification of persons suspected of having an immune deficiency. Additional investigations are needed in order to provide reliable recommendations for the national vaccine program and personalized vaccinations.
\end{abstract}

Key words: diphtheria, tetanus, vaccination, seropositivity, Bulgaria

Corresponding author: Dr. Spaska Stoyneva Lesichkova, MD, Department of Clinical Immunology, University Hospital Alexandrovska, 1, Georgi Sofiiski Street, Bg-1431 Sofia, Ph: (+359)87 8138695 , e-mail: s_lesichkova@abv.bg

RECEIVED: 19 May 2020, ACCEPTED: 26 July 2020 


\section{INTRODUCTION}

$\mathrm{D}$ iphtheria and tetanus vaccines are among the most commonly used vaccines worldwide. Tetanus and diphtheria vaccinations have been available since the 1920 s and most elderly persons have been vaccinated during childhood. In Bulgaria, immunization with diphtheria toxoid has been mandatory since 1951, and after 1967 the combined diphtheria-tetanus-pertusis vaccine began to be used. As a result, only eight cases of tetanus infection have been registered in Bulgaria for the six-year period between 2012 and 2018 [1] and no cases of diphtheria have been reported after 1994 [2]. Despite the arising notion that regular vaccinations over the whole lifetime would be optimal and provide sufficient protection, there is still a lot to be desired in this respect, in particular for people aged 60 years or older, as it has been shown that vaccination frequency in this age group decreases with increasing age [3-7]. Evidence for the effectiveness of vaccination comes from cases in which the disease does not develop after the individual has been exposed to the relevant pathogen. As to rare diseases, vaccination effectiveness is usually assessed by measurements of the titers of specific antibodies in samples from the population of interest. The outcomes of such recurrent analyses could provide valuable information and aid in the evaluation and improvement of national vaccination programs. The available data from multiple reports throughout Europe points to different levels of antibody immune response to diphtheria and tetanus among vaccinated persons, and the differences are more pronounced upon comparisons between age groups [3-10]. In most European countries, antivaccine antibody concentration has been found to decrease with age $[3,5,6,8-10]$. On the other hand, the poor ability to generate post-vaccine synthesis of toxoid and polysaccharide antibodies is one of the defining characteristics of primary immune deficiency (PID) [11]. Therefore, investigations in this direction help to identify individuals suspected of immunodeficiency.
The aim of this study was to determine the concentration of antibodies against diphtheria and tetanus in a group of vaccinated persons of different age groups in the Bulgarian population. We further attempted to correlate the post-vaccine antibody titers with the overall immune competence and the predisposition to more common infectious pathology among our study participants.

\section{MATERIAL AND METHODS}

\section{Clinical material}

This study included 208 participants vaccinated according to the immunization schedule of Bulgaria. Individuals were divided into five age groups: $0-4$ years, 4-6 years, 6-12 years, 12-17 years, 17 years or older (17-66 years) in accordance with the time points for mandatory immunization and re-immunization with tetanus and diphtheria toxoid in Bulgaria (Table 1). Study participants from each age group were further sub-divided into two main categories: healthy $(n=65$, $31.3 \%)$ and symptomatic $(n=143,68.7 \%)$. Symptomatic individuals were defined as persons with a medical history of frequent upper or lower respiratory tract infections or frequent gastro-intestinal infections. We excluded from participation individuals meeting the criteria for primary immunodeficiency or already diagnosed with this condition. Participants in the study (or their parents in case of children and minors) were interviewed in terms of their immunizations and health status and written informed consent was obtained in all cases.

\section{Methods}

Specific antibodies against diphtheria and tetanus toxoid protein antigens were determined in serum samples by indirect enzyme-linked immunosorbent assays (ELISA) using commercial kits (VaccZyme $^{\mathrm{TM}}$ Diphtheria Toxoid IgG ELISA Kit and VaccZymeTM Anti-Tetanus Toxoid IgG ELISA Kit, Binding Site, UK). Assays were performed according to the manufacturer's instructions. Calibrators and internal controls were used for plotting the calibration curve

Table 1. Demographic characteristics of the studied individuals

\begin{tabular}{|c|c|c|c|c|c|}
\hline Age/years & Total number $(\mathbf{n})$ & Males $\mathbf{n}(\%)$ & Females $\mathbf{n}(\%)$ & Healthy & Symptomatic \\
\hline $0-4$ & 53 & $27(51 \%)$ & $26(49 \%)$ & $12(22.6 \%)$ & $41(77.4 \%)$ \\
\hline $4-6$ & 44 & $30(68.2 \%)$ & $14(31.8 \%)$ & $6(13.6 \%)$ & $38(86.4 \%)$ \\
\hline $6-12$ & 44 & $27(61.4 \%)$ & $17(38.6 \%)$ & $15(34.1 \%)$ & $29(65.9 \%)$ \\
\hline $12-17$ & 13 & $7(53.8 \%)$ & $6(46.2 \%)$ & $1(7.7 \%)$ & $12(92.3 \%)$ \\
\hline $17-66$ & 54 & $14(25.9 \%)$ & $40(74.1 \%)$ & $31(57.4 \%)$ & $23(42.6 \%)$ \\
\hline
\end{tabular}


and for quality control, respectively. Antibody concentrations for each patient were then determined by interpolation from the calibration curve.

Based on the kit's instructions and literature data, titers between 0.01 and $0.1 \mathrm{IU} / \mathrm{mL}$ of diphtheria and tetanus antitoxin were considered to represent a minimum protective level (basic protection). Levels higher than $0.1 \mathrm{IU} / \mathrm{mL}$ for diphtheria and $0.15 \mathrm{IU} / \mathrm{mL}$ for tetanus are designated as protective $[12,13]$.

\section{Statistical analysis}

Descriptive analysis was used to report numbers and percentages of individuals. Chi-square test (Fisher's exact test) was applied to compare qualitative variables between groups. Data were analyzed using SPSS v.16.0 (IBM) software. The level of significance was set at $p<0.05$.

\section{RESULTS}

Among the 208 subjects studied, the mean titer of diphtheria antibodies was $0.36 \mathrm{IU} / \mathrm{ml}$ and of tetanus antibodies $-0.80 \mathrm{IU} / \mathrm{mL}$. No gender-related differences were observed. The distribution of serum diphtheria and tetanus antibody titres in the different age groups is shown in Figure 1. According to the WHO guidelines $[12,13]$ sufficient protective levels of DT $(>0.1 \mathrm{IU} / \mathrm{mL})$ and TT $(>0.15 \mathrm{IU} / \mathrm{mL})$ antitoxin were detected in $63.5 \%$ and $85.1 \%$ of all subjects, respectively. Individuals with antibody titers below $0.1 \mathrm{IU} /$ $\mathrm{mL}$ for diphtheria and below $0.15 \mathrm{IU} / \mathrm{mL}$ for tetanus toxoid, were considered insufficiently protected and accounted for $36.5 \%$ and $14.9 \%$ of all subjects, respectively.

IgG antibodies against diphtheria below the minimum protective level $(<0.01 \mathrm{IU} / \mathrm{mL})$ were found in 8 subjects (2 in the age group 0-4 years, 1 in the age groups 4-6 years and 6-12 years, and 4 in the group over 17 years) (Table 2). Pediatric patients with antibodies below the minimum protective level had frequent infections, whereas in adults over 17 years, two were healthy and two had history of recurrent infections.

The age-related dynamics of mean antibody titers against the two toxoids is presented in Figure 2. In the age group 0-4 years, the mean titer of antibodies against DT was $0.56 \mathrm{IU} / \mathrm{ml}$ and against TT - $1.3 \mathrm{lU} /$ $\mathrm{mL}$. Protective levels of antibodies to diphtheria and tetanus toxoid were observed in $79.2 \%$ and $94.3 \%$ of individuals tested, respectively. Insufficient DT protection was observed in one of the healthy and in 10 $(24.4 \%)$ of the symptomatic children (Fig. 3).

All children with insufficient antibody titers against TT belong to the sub-group of frequently ill. In the age between 4 and 6 years, the mean titer of antibodies against DT was $0.32 \mathrm{IU} / \mathrm{mL}$ and against TT $-0.61 \mathrm{IU} /$

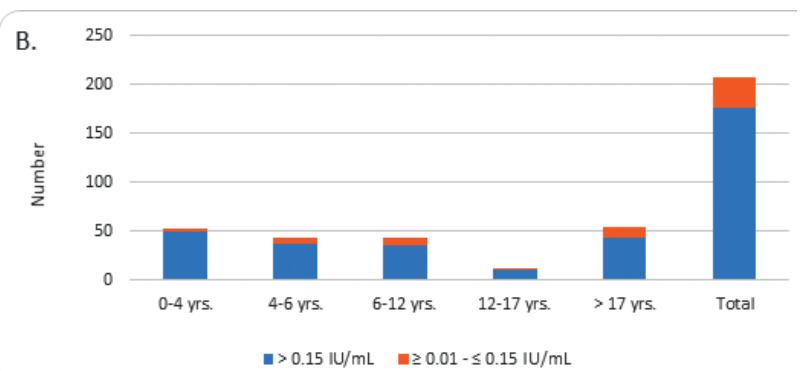

Fig. 1. Distribution of anti-diphtheria (A) and anti-tetanus (B) antibody levels by age groups in 208 individuals from the Bulgarian population

Table 2. Distribution of subjects with reduced/non-protective levels of antibodies against diphtheria (DT) and/ or tetanus (TT) in different age groups

\begin{tabular}{|c|c|c|c|c|c|c|}
\hline \multicolumn{2}{|c|}{ Specific antibodies } & \multicolumn{5}{c|}{ Age group } \\
\hline Anti- DT antibody IU/mL & Anti- TT antibody IU/mL & $\mathbf{0 - 4}$ yr. & $\mathbf{4 - 6}$ yr. & $\mathbf{6 - 1 2}$ yr. & $\mathbf{1 2 - 1 7}$ yr. & $>17$ yr. \\
\hline$<0.1$ & $\mathrm{~N}$ & 7 & 14 & 12 & 2 & 10 \\
\hline $\mathrm{N}$ & $<0.15$ & 1 & 3 & 1 & - & 3 \\
\hline$<0.1$ & $<0.15$ & 2 & 5 & 6 & 2 & 8 \\
\hline$<0.01$ & $<0.15$ & 1 & 1 & 1 & - & 1 \\
\hline$<0.01$ & $\mathrm{~N}$ & 1 & - & - & - & 3 \\
\hline
\end{tabular}

$\mathrm{N}$-protective antibody titers 

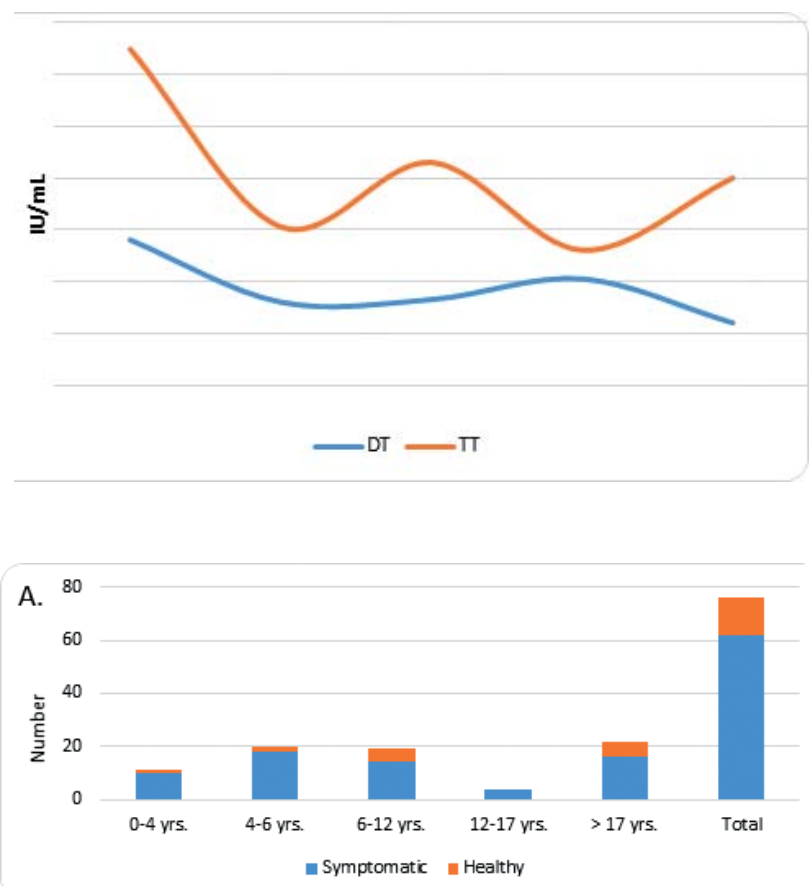

Fig. 2. Age-related dynamics in the mean titers of anti-diphtheria (DT) and anti-tetanus (TT) antibodies

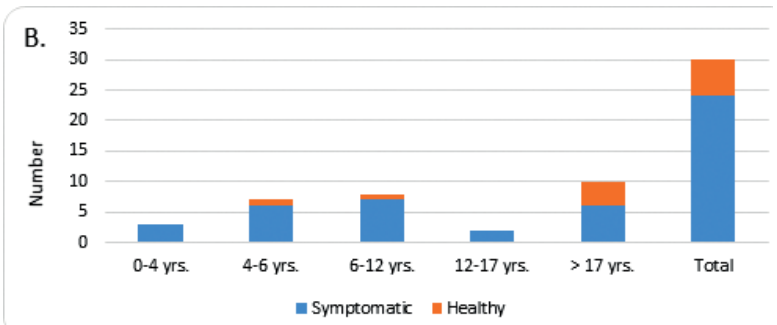

Fig. 3. Distribution by age groups of individuals with insufficient diphtheria (A) and tetanus (B) antitoxin levels

$\mathrm{mL}$. In this group, protective levels against DT were detected in only $54.5 \%$ of children tested. Decreased antibody levels were detected in $33.3 \%$ of the healthy and in $47.4 \%$ of the symptomatic children. Unlike diphtheria toxoid, protective antibody titers against tetanus toxoid were present in $84.1 \%$ of the subjects. In both healthy and symptomatic children, we observed an approximately similar proportion (16.7\% and $15.8 \%$, respectively) of individuals with insufficient protective response to this antigen.

In the age group from 6 to 12 years, the mean titer of antibodies against DT was $0.33 \mathrm{IU} / \mathrm{mL}$ and against TT $-0.86 \mathrm{IU} / \mathrm{ml}$. Similar to the age group 4-6 years, protective levels for DT were observed in $56.8 \%$ and for TT in $81.8 \%$ of all tested children. In $48.3 \%$ of symptomatic individuals the levels of diphtheria antitoxin were below the desired protective thereshold. In contrast to the previous group, decreased antibodies against TT were found in $6.7 \%$ of the healthy and $24.1 \%$ of the symptomatic children. In the age group 12-17 years, the mean anti-DT titers were $0.41 \mathrm{IU} /$ $\mathrm{mL}$ and anti-TT $-0.52 \mathrm{IU} / \mathrm{ml}$. Compared to the previous two groups, we observed a tendency of increase of the proportion of individuals with protective immunity to diphtheria $(69.2 \%)$ and tetanus $(84.6 \%)$. Non-protective values against diphtheria and tetanus were only found in symptomatic subjects, which accounted for $92.3 \%$ of the tested individuals (Fig. 3). However, it should be noted that the number of tested individuals in this age group was very small.
In the adult group (over 17 years) the mean antibody concentration against DT was $0.24 \mathrm{IU} / \mathrm{mL}$ and against TT $-0.80 \mathrm{IU} / \mathrm{ml}$ (Fig. 2). Values above 0.1 $\mathrm{IU} / \mathrm{ml}$ for diphtheria and above $0.15 \mathrm{IU} / \mathrm{ml}$ for tetanus toxoid were detected in $59.3 \%$ and $81.5 \%$ of the subjects, respectively. In this age group, the percentage of subjects with antibody titers below the protective threshold within the symptomatic subgroup was $69.0 \%$ for DT and $26.1 \%$ for TT. The number of subjects with frequent complaints showing insufficient protective levels of antibodies to DT was significantly higher compared to that of healthy individuals $(16 / 23$ symptomatic vs. $6 / 31$ healthy, $p<0.001$, OR $=7.4$ ). Our study on individual antibody profiles showed that in the majority $(58.3 \%)$ of the investigated cases, individuals with insufficient protective titers lacked only antibodies against diphtheria toxoid and in 27 individuals (32.1\%) antibody levels against both antigens were decreased (Table 2).

\section{DISCUSSION}

The basic vaccination regimen for diphtheria and tetanus is similar in different regions of the world, with variation mainly in the number and timing of booster doses [14]. The diphtheria and tetanus vaccination schedule in Bulgaria includes a total of 7 doses: an initial course of 3 doses in the first four months of life and one dose in the second year, with boosters aged 6, 12 and 17 years. Tetanus and diphtheria immuni- 
zation is given at the age of 25 and every 10 years thereafter. Post-exposure tetanus prophylaxis is given to adult patients with 1 dose of tetanus toxoid.

In this study, we investigated the age-related dynamics in post-vaccine antibody immune response to diphtheria and tetanus toxoid in the Bulgarian population. We found a different response to the antigens tested. The tetanus toxoid is widely considered to be highly immunogenic and results in a good immune response [14]. Our results showed that the majority $(85.1 \%)$ of the individuals tested had protective antibody titers against tetanus. The highest rate of protective antibody titers was observed in the age group 0-4 years $(94.3 \%)$, probably related to vaccination schedule. Although a gradual decrease in protection was observed at 4-6 years of age $(84.1 \%)$ and between 6 and 12 years (81.8\%), and a slight increase of subjects with protective values $(84.6 \%)$ was noted in the next age cohort (12-17 years), the proportion of protected children was high. These insignificant variations may be partly due to testing each individual in the respective group at a different time point after their last immunization or to the relative small sample size of individuals aged 12-17 years. It should be also noted that $81.5 \%$ of adults (17 years or older) also showed a good level of antibody protection against tetanus. Our data and conclusions agree well with the outcomes of a cross-sectional study in Austria demonstrating a decline of tetanus-specific antibody concentrations with increasing time since the last vaccination and lower antibody concentrations in elderly people [15]. In contrast, a study in Poland found that the proportion of children with a protective response gradually increases with age with the lowest levels observed in the youngest subgroup [16]. However, the study of Postfay-Barbe et al. demonstrated that after receiving 3 doses of early childhood vaccination in Cameroon, only $55 \%$ of adolescents were able to produce a minimum protective antibody level of 0.1 $\mathrm{IU} / \mathrm{ml}$ upon administration of a single booster dose of tetanus toxoid [17]. The authors explain these findings with the immaturity of the adaptive immune response in infants that may limit a long-term immune memory in the first months of life. The study of Schauer et al. in Germany in a large cohort of age-stratified healthy individuals revealed that $14 \%$ of the tested adult samples from 20 to 61 years of age had anti-tetanus antibody levels below the protective limit of $0.15 \mathrm{IU} / \mathrm{ml}$ [18]. A slightly higher percentage $(18.5 \%)$ of insufficiently protected individuals was found in our adult group over 17 years old. However, in a Polish study, regardless of age, the proportion of unprotected persons did not exceed $10 \%[16]$.
With regards to the diphtheria protective immune response, although the age-related differences follow the same downward trend as those for tetanus, they are much more pronounced between different age groups. The highest rate of protection (79.2\%) were observed in the age of 0-4 years. Approximately $55-57 \%$ of the children between the ages of 4 and 11 years have antibodies titers above $0.1 \mathrm{IU} / \mathrm{mL}$. Between 12 and 17 years an increase of protected subjects to $69.2 \%$ was noticed, followed by a decrease to $59.3 \%$ in adults. A high proportion of children without sufficient diphtheria protection (30\%) was reported in Poland [16]. Although the authors consider this an exception, we also observed a similar rate of insufficient protection against diphtheria (36\% of individuals tested across all age groups). Overall, the percentage of children with protective diphtheria antibody levels is lower than the percentage of children with protective tetanus antibody levels, and this trend is evident both in Bulgaria as well as in other European countries [9, $10,19,20]$. Despite the validity of this general trend, different countries do have different rates of protected individuals among age groups, which might be due to differing vaccine schedules and epidemiological factors $[3,19]$. A study in Iran demonstrated very high percentages of infants with protective antibody levels (> $1.0 \mathrm{lU} / \mathrm{ml}$ ) against diphtheria (98\%) and tetanus (100\%) 2-4 weeks after the third dose of primary immunizations [21]. Maintenance of high levels of protection against diphtheria (81\%) and tetanus (96\%) was shown in Austrian children ages 4 to 8 years who had received 4 doses of vaccine [15]. In a study of healthy Slovak adults Petras et al. detected protective levels of tetanus antibodies in all individuals aged 10-16 years after a booster dose vaccine while only $88.1 \%$ of the investigated subjects had high levels of protection (titers $\geq 0.1 \mathrm{IU} / \mathrm{mL}$ ) against diphtheria [10]. Their results support the observations of stronger immune response against tetanus in comparison to diphtheria which is evident in our study as well.

When we compared healthy participants and those with more frequent infections, it was found that in almost all age groups the percentage of insufficient protection against both antigens was higher among frequently ill subjects (except for the antibody titers to tetanus in the age group 4-6 years). The difference reaches statistical significance for diphtheria antitoxin $(p=0.002 ; O R=2.57)$. It is interesting to note that $77.1 \%$ of the individuals having low antibody titers against the highly immunogenic tetanus toxoid, also had low levels of diphtheria antibodies. The percentage of cases with simultaneous insufficient protection to both antigens was reported to be higher in Poland $(95 \%)$ [16]. 
The generation of an efficient antibody response to antigenic challenges delivered via vaccination provides an essential insight into the immune status of children and adults suspected of having primary antibody deficiencies and other types of deficiencies. Several studies demonstrated impaired immune response to immunization in children with hypogammaglobulinemia [11, 22, 23]. Although the measurement of post-vaccination antibody levels is an integral part of the immunological evaluation of patients with suspected PID, the results should be interpreted with caution. In frequent antibody production defects in children, such as transient hypogammaglobulinemia of infancy, IgG subclass deficiency, IgA deficiency and specific anti-polysaccharide deficiency, the antibody response to protein and protein-conjugated vaccines is typically preserved. Moreover, in a subset of pediatric patients with low antibody levels and impaired response to vaccines, a lack of evidence for specific diagnosis of PID may indicate a physiological delay of the immune response [23].

In our study, patients with PID were excluded based on standard immunological testing and clinical criteria. The IgG, IgM and IgA serum levels of the studied individuals were within the reference ranges (data not shown). Further studies and analysis of immunoglobulin subclasses and the use of some specific cellular functional assays in symptomatic individuals with insufficient antibody titers against diphtheria and tetanus are warranted in order to identify secondary abnormalities in the immune response, which could eventually explain the susceptibility to frequent pathology.

The data from this study showed that anti-tetanus antibodies exhibit relatively more stable levels during life, with the percentage of protected subjects beyond 4 years of age varying between 81 and $86 \%$. Of greater concern is the trend of frequent insufficient immune response against diphtheria in the first years of life, which deepens progressively during adulthood. This, along with the inadequate immunization coverage and a number of disadvantageous socioeconomically factors, puts a portion of the population at risk of developing vaccine-preventable infections in the 21 st century. Compared to the group of healthy individuals, people with frequent infectious display significantly higher rates of insufficient protection against diphteheria and tetanus and this requires a search for secondary immune deficiency or discrete primary disorders in the immune response in the latter group of participants. Measures to improve protection should include a long-term perspective on vaccination, more education to increase awareness and compliance with vaccination guidelines.
In conclusion, the data generated by the present study provides information on the level of immunity to diphtheria and tetanus among vaccinated children and adults in Bulgaria and allows for the identification of persons suspected of having an immune deficiency. A disadvantage of the present study is the insufficient number of individuals in some age groups, which does not allow for more generalized conclusion. Additional investigations are needed in order to provide reliable recommendations for the national vaccine program and personalized vaccinations.

Disclosure summary: The authors have nothing to disclose.

Acknowledgments: This study was supported by a grant D-99/23.04.2019 from the Medical University, Sofia. We thank Stanislava Popova from DZNE, Helmholtz Association, Dresden, Germany for language editing and comments on the manuscript.

\section{REFERENCES}

1. Analysis of the implementation of the activities for immunoprophylaxis in Bulgaria in 2018. Epidemiology Department, National Center for Infectious and parasitic disease, Sofia, Bulgaria.

2. European immunization week, 23-29 APRIL 2018, WHO, Communications package, Vaccineswork.

3. Weinberger B, Keller M, Putzer C et al. Protection against Tetanus and Diphtheria in Europe: The impact of age, gender and country of origin based on data from the MARK-AGE Study. Exp Gerontol. 2018; 105:109-112. doi: 10.1016/j. exger.2017.08.037.

4. Bayas JM, Vilella A, Bertran MJ et al. Immunogenicity and reactogenicity of the adult tetanus-diphtheria vaccine. How many doses are necessary? Epidemiol Infect 2001; 127:451-460.

5. Launay O, Toneatti C, Bernede $\mathrm{C}$ et al. Antibodies to tetanus, diphtheria and pertussis among healthy adults vaccinated according to the French vaccination recommendations. Hum Vaccin 2009; 5:341-346.

6. Kwetkat A, Lehmann T, Weinberger S, Schelling J. Analysis of vaccination data of patients aged 60 years and older from Bavaria and Thuringia. Z Gerontol Geriatr 2016; 49:126-131.

7. Kanitz EE, Wu LA, Giambi C. Variation in adult vaccination policies across Europe: an overview from VENICE network on vaccine recommendations, funding and coverage. Vaccine 2012; 30:5222-5228.

8. Hainz $U$, Jenewein $B$, Asch $E$ et al. Insufficient protection for healthy elderly adults by tetanus and TBE vaccines. Vaccine 2005; 23:3232-3235.

9. Zasada A, Rastawicki W, Rokosz N, et al. Seroprevalence of diphtheria toxoid IgG antibodies in children, adolescents and adults in Poland. BMC Infect Dis 2013;13:551. doi: 10.1186/1471-2334-13-551.

10. Petráš $M$, Oleár $\mathrm{V}$, Molitorisová $\mathrm{M}$ et al. Factors Influencing Persistence of Diphtheria Immunity and Immune Response 
to a Booster Dose in Healthy Slovak Adults. Vaccines (Basel). 2019; 7(4). pii: E139. doi: 10.3390/vaccines7040139.

11. Szczawinska-Poplonyk A, Breborowicz A, Samara H et al. Impaired antigen-specific immune response to vaccines in children with antibody production defects. Clin Vaccine Immunol 2015,22:875-882.doi:10.1128/CVI.00148-15.

12. World Health Organization. (2009)?. Immunological basis for immunization: module 2: diphtheria-update 2009, Update 2009. World Health Organization. https://apps.who.int/iris/ handle/10665/44094

13. The immunological basis for immunization series: module 3 : tetanus. Geneva: World Health Organization; 2018 (Immunological basis for immunization series; module 3 ). Licence: CC BY-NC-SA 3.0 IGO.

14. Advisory Committee on Immunization Practices. Updated recommendations for use of tetanus toxoid, diphtheria toxoid and acellular pertussis vaccine from the Advisory Committee on Immunization Practices 2010. MMWR Morb Mortal Wkly Rep 2011; 60:13-15.

15. Paulke-Korinek M, Fischmeister G, Grac A et al. Peristence of antibodies in 4-8 year old Austrian children after vaccination with hexavalent DTaP-HBV-IPV/Hib and MMR vaccines. Vaccine 2011; 29:5130-5136.

16. Gowin E, Wysockib J, Kaluzna E et al. Does vaccination ensure protection? Assessing diphtheria and tetanus antibody levels in a population of healthy children. A cross-sectional study. Medicine 2016; 95:49(e5571), dx.doi.org/10.1097/ MD. 0000000000005571.

17. Postfay-Barbe KM, Kobela M, Sottas $C$ et al. Frequent failure of adolescent booster response to tetanus toxoid despite infant immunization: waning of infancy-induced immune memory? Vaccine 2010; 28:4356-4361. doi:10.1016/j.vaccine.2010.04.060.

18. Schauer $\mathrm{U}$, Stemberg $\mathrm{F}$, Rieger $\mathrm{CH}$ et al. Levels of antibodies specific to tetanus toxoid, Haemophilus influenzae type b, and pneumococcal capsular polysaccharide in healthy children and adults. Clin Diagn Lab Immunol. 2003; 10(2):202-207.

19. Edmunds WJ, Pebody RG, Aggerback H, et al. The seroepidemiology of diphtheria in Western Europe. ESEN Project. European Sero-Epidemiology Network. Epidemiol Infect 2000; 125:113-125.

20. Walory J, Grzesiowski P, Hryniewicz W. The prevalence of diphtheria immunity in healthy population in Poland. Epidemiol Infect 2001; 126:225-230.

21. Zarei S, Jeddi-Tehrani M, Akhondi MM et al. Primary immunization with a triple diphtheria-tetanus-whole cell pertussis vaccine in Iranian infants: an analysis of antibody response. Iran J Allergy Asthma Immunol 2009; 8:85-93.

22. Dorsey MJ, Orange JS. Impaired specific antibody response and increased B-cell population in transient hypogammaglobulinemia of infancy. Ann Allergy Asthma Immunol 2006; 97:590-595.

23. Orange JS, Ballow M, Stiehm ER et al. Use and interpretation of diagnostic vaccination in primary immunodeficiency: a working group report of the Basic and Clinical Immunology Interest Section of the American Academy of Allergy, Asthma \& Immunology. J Allergy Clin Immunol. 2012 Sep;130(3 Suppl):S1-24. doi: 10.1016/j.jaci.2012.07.002. 